\title{
Filigrane
}

Écoutes psychothérapiques

\section{Pour une métapsychologie de l'espace. Une troisième topique}

\section{Lise Marceau}

Volume 22, numéro 2, automne 2013

Psychanalyse et temporalités II

URI : https://id.erudit.org/iderudit/1022558ar

DOI : https://doi.org/10.7202/1022558ar

Aller au sommaire du numéro

Éditeur(s)

Revue Santé mentale au Québec

ISSN

1192-1412 (imprimé)

1911-4656 (numérique)

Découvrir la revue

\section{Citer cet article}

Marceau, L. (2013). Pour une métapsychologie de l'espace.

Une troisième topique. Filigrane, 22(2), 89-102.

https://doi.org/10.7202/1022558ar

\section{Résumé de l'article}

Le projet d'une troisième topique permet de pallier aux insuffisances de la métapsychologie freudienne, soit le manque d'importance accordée à la réponse de l'objet dans la constitution du psychisme humain. Elle permet aussi de déterminer les conditions de passage de l'unité duelle primordiale à l'unité individuelle intrapsychique, et l'avènement d'un espace dit transitionnel, déjà élaboré par Winnicott. Espace transitionnel, lieu de la naissance du sujet qui nous fera évoquer les pathologies du transitionnel ou des problématiques narcissiques-identitaires. La troisième topique est donc abordée ici comme un outil qui permet d'élaborer une métapsychologie de l'espace, ainsi que de conceptualiser la clinique des états limites qui interroge la méthode psychanalytique quant à ses fondements. Nous revisiterons les concepts clé utilisés par Reid, tel que le Soi, l'hallucinatoire, le travail du Négatif et la transitionnalité. Pour éclairer notre parcours dans cet espace créé/découvert par Winnicott, nous nous servirons aussi des lumières de Pontalis, Ogden et quelques autres.
Ce document est protégé par la loi sur le droit d'auteur. L'utilisation des services d’Érudit (y compris la reproduction) est assujettie à sa politique d'utilisation que vous pouvez consulter en ligne.

https://apropos.erudit.org/fr/usagers/politique-dutilisation/ 


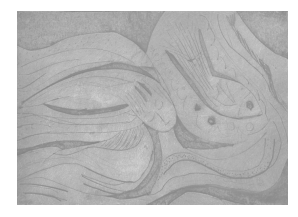

\section{Pour une métapsychologie de l'espace. Une troisième topique ${ }^{1}$}

\section{Lise Marceau}

Le projet d'une troisième topique permet de pallier aux insuffisances de la métapsychologie freudienne, soit le manque d'importance accordée à la réponse de l'objet dans la constitution du psychisme humain. Elle permet aussi de déterminer les conditions de passage de l'unité duelle primordiale à l'unité individuelle intrapsychique, et l'avènement d'un espace dit transitionnel, déjà élaboré par Winnicott. Espace transitionnel, lieu de la naissance du sujet qui nous fera évoquer les pathologies du transitionnel ou des problématiques narcissiquesidentitaires. La troisième topique est donc abordée ici comme un outil qui permet d'élaborer une métapsychologie de l'espace, ainsi que de conceptualiser la clinique des états limites qui interroge la méthode psychanalytique quant à ses fondements. Nous revisiterons les concepts clé utilisés par Reid, tel que le Soi, I'hallucinatoire, le travail du Négatif et la transitionnalité. Pour éclairer notre parcours dans cet espace créé/découvert par Winnicott, nous nous servirons aussi des lumières de Pontalis, Ogden et quelques autres.

'idée d'une troisième topique n'est pas nouvelle. Déjà Reid en 1996, _ Green (1982, 1983, 1990), Desjours (1986), Racamier (1992), Bercherie (2000), Cahn (2002) et d'autres, l'avaient proposée. À Catherine Chabert qui n'en voyait pas la nécessité et craignait un infléchissement de la méthode, Bernard Brusset répondait en 2005 au congrès de psychanalyse des langues romanes tenu au Portugal, que peu importait qu'on l'appela troisième topique ou autrement. Ce qui est important, c'est l'élaboration d'une théorie ou d'un outil ancré dans la clinique actuelle des états limites, qui permette à la méthode psychanalytique d'être éclairée quant à ses réussites ou ses échecs (Brusset, 2005). Un outil qui permette de penser aux frontières de l'analysable: état limite ou problématique narcissique-identitaire (Roussillon) d'un sujet qui ne trouve pas sa place par rapport aux autres, ou que les autres n'arrivent pas à mettre à sa place. Un sujet naviguant entre 
fusion, confusion et sensation, plus ou moins soutenable, de vide ou de chaos.

\section{Les deux topiques freudiennes}

D'entrée de jeu, situons les deux topiques freudiennes qui seraient un point d'arrivée par rapport à la topique troisième qui elle, serait un point de départ. D'abord le terme topique signifie théorie des lieux, et nous vient de l'antiquité grecque. Si l'observation des phénomènes hypnotiques et hystériques fut le contexte qui permit à Freud la découverte de l'inconscient, le contexte scientifique de la théorie anatomophysiologique des localisations cérébrales lui permit de d'esquisser l'idée d'une topique psychique. Bien qu'elle existe en filigrane dans la pensée de l'Esquisse, la première conception topique de l'appareil psychique n'est présentée que dans le chapitre 7 de l'Interprétation des rêves, quelques années plus tard. Elle distingue trois « régions » ou systèmes: le conscient, le préconscient et l'inconscient. Entre chacun de ces systèmes, Freud situe des censures qui contrôlent le passage de l'un à l'autre. Les systèmes peuvent être parcourus dans une direction normale progrédiente ou régrédiente. Freud parlera alors de «régression topique » illustrée par le phénomène du rêve, où les pensées régressent du côté des images, images hallucinatoires au plus proche de la perception, à l'origine du parcours de l'excitation. «Soutenue par le paradigme de l'hystérie, observe Chabert, cette topique inclut la distinction entre pulsion d'autoconservation et pulsion sexuelle et s'engage dans la voie du plaisir, de la satisfaction des désirs et de la guérison» (Chabert 2007,2).

Avec la découverte du narcissisme (Freud, 1914), cette première « distribution spatiale» s'avère insuffisante. À partir de 1920, Freud élabore donc une autre conception de l'appareil psychique, la seconde topique, avec ses trois instances - le ça, le moi et le surmoi —, laquelle se structure sous le signe de la conflictualité. Sous-tendue cette fois par la référence au narcissisme et à la mélancolie, elle distingue pulsion de vie et pulsion de mort. «Elle s'engage dans la voie de la douleur [la perte] la compulsion de répétition et le refus de guérir» (Chabert 2007, 2). Il faut souligner ici, que le moi étant constitué, la limite entre dedans et dehors est déjà établie. Entre les deux topiques, une dialectique s'établit, sans cesse présente et active. Le conflit et la perte se conjuguent à l'infini et dans tous les registres : topographique, économique et dynamique. 


\section{La troisième topique}

Reprenons la question, pourquoi une troisième topique? Pour pallier aux insuffisances de la métapsychologie freudienne, dit en substance Wilfrid Reid, soit le manque d'importance accordé à la réponse de l'objet dans la constitution du psychisme. En effet, il aura fallu attendre Winnicott avec les concepts de «holding» et de "mère suffisamment bonne», et Bion avec les concepts de "relation contenant-contenu ", "capacité de rêverie de la mère", et «identification projective normale», pour que le rôle de l'objet dans le développement puisse être conceptualisé.

Une troisième topique, ajoute Reid, pour déterminer les conditions de passage de l'unité duelle primordiale, soit l'ensemble individu/environnement, à l'unité individuelle intrapsychique, passage qu'il nomme " travail de monadisation ». Reid définit la monade freudienne comme «l'auto organisation de la conflictualité inconsciente intrapsychique» (Reid, 1997, 1324). Dans cette idée de transition ou de passage, il faut considérer le mouvement en lui-même, un mouvement psychique qui est générateur d'espace, un nouvel espace, en l'occurrence, où le $\mathrm{Je}(\mathrm{u})$ devient possible. Une énergie potentielle et cinétique en quelque sorte, qui permet de concevoir la pulsion comme impulsion, élan, et non plus seulement comme effraction (Reid, 2006, 1548). Un Je (u) qui n'est jamais définitif, qu'il faut sans cesse recréer, rejouer. Le concept d'un ensemble individu/environnement, tel que promu par Winnicott, servira donc d'assise au modèle de la troisième topique qui comportera deux instances, celle du Soi et celle de l'objet, et se théorisera autour de quatre axes majeurs: l'hallucinatoire, la transitionnalité, la double limite et le travail du négatif.

\section{Le Soi}

D'abord, demandons-nous ce qu'il faut entendre par le terme «Soi». Il semble qu'il recouvre la question de l'être et du sentiment d'être vivant. Pontalis raconte qu'une traductrice avait demandait à Winnicott de préciser la différence entre moi (ego) et Soi (self). "Après avoir avoué son embarras, Winnicott répondit que l'utilisateur du terme soi (self) ne se situe pas sur le même registre que l'utilisateur du terme moi (ego)» (Pontalis, 1977,186). Le moi se présente "comme une image devant le miroir», ramassis d'identifications, «espace clos, enchâssé » entre l'espace du ça, du surmoi et du monde extérieur. Il se distingue du Soi qui est «dans l'espace psychique, le représentant du vivant», "espace ouvert sur l'environnement qui le nourrit [...] et qu'en retour il crée». Au bout du compte, dit Pontalis, ce serait «se poser et être reconnu comme étant» (187). 
Winnicott distingue aussi le faux Soi du vrai Soi. Pontalis suggère d'entendre cette distinction comme une double polarité. Le Soi devient alors le résultat du «difficile équilibre à édifier entre les forces de maturation et les apports de l'environnement. » Conceptualisé de cette manière, le faux Soi a pour tâche de protéger le vrai Soi, et permet de saisir « une des vues les plus originales de Winnicott soit le droit de pas être découvert, le besoin de ne pas communiquer [...] [qui] révèle que l'individu se sent réel dans la communication secrète qu'il entretient avec ce qu'il y a en lui de plus subjectif» (188). "Chez l'artiste, écrit Winnicott, coexistent deux courants contraires. [...] Le besoin pressant de communiquer et le besoin encore plus pressant de ne pas être trouvé» (Winnicott, 1970, 158-160). «Être caché est une joie mais c'est une catastrophe de ne pas être trouvé» (Pontalis, 1977, 180). Pour rendre compte de la présence ou de l'absence du vivant dans la vie psychique, Wilfrid Reid souligne que Winnicott a introduit deux nouveaux concepts spécifiques de sa métapsychologie, soit l'Être et le Faire. Il désignera « un faire par impulsion issu de l'être [...] par opposition à un faire par réaction, par le non établissement de l'être, qui permettra ou non à l'individu de devenir l'auteur de la poussée pulsionnelle dans la représentation inconsciente» (Reid, 2006, 1547).

Rappelons ici que l'œuvre de Winnicott se situe dans le contexte d'un débat entre les freudiens classiques et les kleiniens, dans le contexte d'une pratique de la psychanalyse qui présentait celle-ci comme une idéologie, voire une machine à interpréter ce que dit et éprouve le patient. La pensée de Winnicott a pu surgir en réaction à "L'idée de complaisance, de soumission à l'environnement, d'un sujet littéralement capté dans le système de l'autre qu'il s'agisse de la mère ou de l'analyste» (Pontalis, 1977, 188). En même temps, l'œuvre de Winnicott est le produit indéniable de l'élaboration d'une pensée créatrice. Il écrit concernant celle-ci: «En fait, je glane une chose et une autre ça et là, me penche sur l'expérience clinique, élabore mes propres théories et puis, tout à la fin, je cherche à voir ce que j'ai volé et où. Peut-être cette méthode en vaut-elle une autre» (Winnicott, 1945, 57).

\section{L'hallucinatoire}

En 1897, l'abandon par Freud de sa Neurotica correspond, selon Reid, à la découverte de l'hallucinatoire, qu'il définit comme «la coalescence du dedans et du dehors", "l'expression première de la mise en mouvement, de la motricité de la psyché» (Reid, 2006, 1549). Bien que Freud ait déjà utilisé le terme, il ne s'y est pas vraiment arrêté. André Green, en 1977, fut le premier 
à élaborer ce concept dans son texte sur l'hallucination négative (Green, 1993). César et Sarah Botella l'ont repris. Pour eux, l'hallucinatoire comprend "le représentant motionnel de la pulsion», «un état de qualité psychique potentiellement permanent, formé de continuité, d'équivalence, d'indistinction représentation-perception; où le perçu et le percevant, le figuré et le figurant ne font qu'un. Il serait de l'essence du rêve, tout en existant indépendamment de lui. Plus près du pôle perceptif et moteur que de la conscience et de la pensée [...] » (Botella et Botella, 2002, 723-725). Au quotidien, "il sous-tend la figurabilité diurne de la même manière qu'il soustend le rêve nocturne; il participe à la vivacité des souvenirs de même qu'il contribue à éveiller le sentiment d'évidence, de conviction. [...] l'hallucinatoire, comme l'inconscient, représente dans le psychisme un véritable pôle d'attraction, une violente aspiration du désir à obtenir satisfaction par le plus court chemin, court-circuitant la fonction de représentation. [...] Il reviendrait au processus primaires le rôle de tempérer, d'harmoniser cette double attraction» (Botella et Botella, 1990, 82-83). Pour appréhender l'hallucinatoire, disent-ils, il faut s'affranchir du carcan de la spatio-temporalité, ce qui n'est pas évident. Les Botella préconisent un «au delà de l'abandon de toute représentation-but par l'analyste, l'acception plus loin que l'attention flottante, d'un état de passivité du moi, de relâchement [...] des mailles du réseau associatif» (Botella et Botella, 1990, 64). Cela pourra se manifester cliniquement, selon Reid, «dans la manière de sentir, de penser et de dire de l'analyste», enfin de se «libérer de la tyrannie des contenus» (Reid 2005, 37), ou bien, « un état sans désir et sans mémoire », préconisé par Bion.

\section{La négativation de l'hallucinatoire}

Pour Wilfrid Reid, «la négativation de l'hallucinatoire, assurera au plan économique, la délimitation des territoires respectifs des systèmes inconscient et préconscient de sorte que la réalité de pensée cesse d'être équivalente à la présence de la chose dans la réalité extérieure». Mais comment pourra s'accomplir cette négativation? "La psyché, poursuit-il, devra générer une force proportionnelle antagoniste au mouvement hallucinatoire» (Reid, 2005, 36-37). Mais encore, qu'est-ce qui motivera la psyché à ce moment-là, à générer une telle contre-force? Où trouvera-t-elle son énergie puisque la réalisation hallucinatoire du désir permet d'éviter la détresse liée à l'absence de l'objet et constitue une première tentative de liaison? "Que l'état de détresse est un état extrême de détresse, par l'échec du psychisme à se représenter la détresse. [...] L'investissement de la répétition hallucinatoire d'une 
perception (qui en appelle au souvenir de la trace) est déjà une première liaison anti-traumatique, une explication, un début d'élaboration » (Botella et Botella, 1990,71).

Alors pourquoi et comment s'affranchir de l'hallucinatoire? Si Freud répond que cela n'assouvit pas la faim, Reid répond que «ce travail psychique sera promu par l'environnement dont le rôle paradoxal consistera à favoriser un processus d'absentification de cet environnement, facilitant du même mouvement, la création d'une interface différenciant l'objet du dehors et l'objet du dedans » (Reid, 2005, 36-37). Ainsi, la négativation de l'hallucinatoire ouvre l'espace transitionnel au fondement d'une métapsychologie de l'espace. Elle dépend donc de la capacité négative de l'environnement, ou de «la constitution progressive de l'absence ». «La mère absente fait notre intérieur et notre "vrai soi" est la relation maintenue vivante, avec cette absence, sans quoi le sentiment d'être et de vivre fait défaut» (Pontalis, cité par Reid, 1997, 1333).

Un bébé ça n'existe pas, rappelle Reid à la suite de Winnicott. Ce qui existe c'est l'unité mère-bébé. Avec Green, nous soutenons qu'une mère et un enfant ça n'existe pas davantage sans père. C'est la représentation du père dans la psyché de la mère, qui soutient sa capacité négative, capacité de silence et de non empiètement. Le père, "good-enough », défini comme représentant de la loi, de l'interdit de l'inceste et du meurtre, représentant d'une loi fondamentale entre les hommes, figure ce Tiers qui vient « rompre les charmes » (Leclaire, 1981) et assurer la nécessaire désillusion donnant accès à l'altérité. Cette capacité négative de la mère est aussi soutenue par son désir pour l'homme dans le père, son désir pour un Pas-Moi qui viendra "protéger l'enfant d'un excès de passion» (Green, 1980).

\section{L'espace transitionnel} L'objet trouvé-créé

Voyons de plus près ce qu'il en est. Au début, rappelle Reid, c'est le règne de l'ensemble individu-environnement, la dyade soi-objet, plus simplement, infans/environnement. Il s'agit de la mère-environnement qui s'identifie totalement à son bébé, tout en restant elle-même pour assurer la fonction de holding. Les deux, mère et infans, constituent à ce moment-là une unité, une unité duelle, une organisation qui contient les traits les plus primitifs et les plus matures du développement d'un individu; donc un soi qui s'ignore et un objet ignoré dans son extériorité, dont le soi dépend entièrement sans le savoir. Objet maternel primaire, mère-environnement qui par sa capacité 
négative de s'effacer comme objet, de s'absenter tout étant présente, à la bonne distance et au bon moment, permettra la création de l'objet subjectif. La mère, dit Winnicott, "place le sein réel juste là où l'enfant est prêt à le créer et au bon moment. » "Il y a chevauchement entre l'apport de la mère et ce que l'enfant peut concevoir» (Winnicott, 1971, 21-22). C'est le fameux paradoxe de l'objet trouvé/créé.

«La mère environnement est un espace avant d'être un objet", "un entour indivis » (Reid, 2006, 1546-1547) ou encore une «ambiance» (Green 1993,369). On pourrait dire aussi, un espace-temps puisque «le délai de la réponse maternelle est capital pour assurer une expérience de satisfaction qui ne soit ni aliénante ni destructrice» (Green, 1993, 370).

La mère-environnement doit protéger l'infans de toute excitation pulsionnelle qu'il ne pourrait contenir, qui risquerait de le bouleverser et de déclencher en lui un reflexe d'agrippement ou une maturité précoce. Elle le protège de se sentir séparé, mais sans empêcher qu'il fasse un jour l'expérience de la séparation qui lui donnera le sentiment d'être lui-même, auteur de son expérience. Elle protège son sommeil, si l'on peut dire, mais sans l'empêcher de se réveiller.

Avec la maturation neurobiologique, musculaire et cognitive de l'enfant, la mère-environnement va s'adapter aux nouveaux besoins de l'enfant et à sa capacité croissante d'autonomie. Après avoir donné à l'enfant la possibilité de l'illusion, le rôle de la mère participe désormais à une désillusion progressive, c'est-à-dire un sevrage progressif du holding. Phase de sevrage qui correspond à l'appropriation par l'enfant de cette unité mère-enfant qu'Ogden appelle «matrice psychologique» (Ogden, 1985b, 355). Ce faisant, l'enfant développe peu à peu la capacité d'être seul, il développe l'aptitude "à générer l'espace», "a state of being». «Puisque la mère participe de façon effacée à la création de cet espace, écrit Ogden, quand l'enfant sain est seul, il l'est toujours en présence de la mère » (Ogden, 1985b, 356). La mère vit une expérience avec l'enfant dans laquelle elle continue de s'offrir comme objet qui peut être expérimenté par l'enfant comme sa création, puisque que tout se passe selon ses besoins, et en même temps, elle peut être expérimentée comme une découverte, puisqu'il ne l'avait pas prévue, ni attendue; un évènement avec une qualité d'altérité dans un monde externe, distinct du soi. Un évènement pulsionnel en l'occurrence, ne serait-ce que par la curiosité qu'il suscite. La pulsion est désormais conçue non plus comme effraction, mais comme impulsion, comme élan, tel que nous l'avons rapporté plus haut. 
L'expérience de «l'objet trouvé/créé» sous-tend chez l'infans le sentiment d'omnipotence et de pensée magique. Reid définit le sentiment d'omnipotence comme étant une hallucination du non désir, puisque «His majesty the baby» est à l'abri de la sensation du manque. La question n'est plus de savoir comment l'enfant va se développer, mais plutôt comment il lui sera possible d'émerger de la dyade sans traumatisme. C'est ici qu'intervient l'objet transitionnel.

\section{L'objet et l'espace transitionnel}

L'objet transitionnel est ce nom donné par Winnicott à la doudou que nous connaissons tous, petite couverture ou bout de tissu que l'enfant serre contre lui, suçote, et dont il ne peut se passer, particulièrement au moment de l'endormissement. Il est un calmant. «Une défense contre l'angoisse de type dépressif», "la première possession, de quelque chose qui n'est pas moi ». Il se situe entre la mère et l'enfant, à la fois le sein et à la fois distinct de ce sein. "Il est à la fois l'enfant, - une extension omnipotente de luimême et en même temps il n'est pas l'enfant, — un objet découvert en dehors de son contrôle omnipotent. La question n'est jamais posée: cet objet l'ai-je créé ou l'ai-je découvert?» (Winnicott, 1971, 8).

Ainsi, l'espace transitionnel prend son origine et se déploie entre le subjectif et l'objectif, entre le dehors et le dedans, ni intérieur ni extérieur, mais un espace entre les deux auquel contribuent la réalité extérieure et la vie intérieure, soit la mère et l'enfant. L'enfant et la mère sont un, côté fantasme, et en même temps ils sont deux, côté réalité (Ogden, 1985a, 131). Le statut du transitionnel est donc de l'ordre du paradoxe, et Winnicott insiste pour que «le paradoxe soit accepté, toléré et non résolu. » Cet espace ne doit jamais être «actuel», sans quoi l'enfant pourrait faire l'expérience d'un vide traumatique, non représentable pour lui avec les angoisses, " agonies primitives » ou «terreurs sans nom », que l'on sait. L'objet transitionnel est donc un symbole de la séparation dans l'unité et de l'union dans la séparation. Il témoigne de la possibilité de ne pas résoudre le paradoxe, et aussi du développement de la capacité de maintenir un processus dialectique entre des termes opposés. En effet, si on y regarde de près, avec Ogden, l'acquisition de cette capacité dialectique implique la transformation de l'unité en une interaction dynamique entre trois entités différenciées: le symbole (l'objet transitionnel), le symbolisé (l'union dans la séparation), et un sujet interprétant. Cette transformation d'une unité en trois termes coïncide, poursuit Ogden, avec la transformation de l'unité mère-enfant en trois unités distinctes: la mère- 
enfant, la mère et l'enfant, et l'enfant observateur des deux. Au moment de cette différenciation, non seulement la mère et l'enfant sont créés comme objets, mais en plus, l'enfant est créé comme sujet. Cette acquisition d'une pensée dialectique est essentielle, puisqu'elle signifie la capacité d'élaborer une signification personnelle qui est représentée par des symboles issus de la subjectivité. L'enfant devient ainsi maintenant le créateur et l'interprétant de ses symboles (Ogden, 1985a, 131-132). Cette différenciation crée la possibilité d'une triangularité dans laquelle un espace est créé. C'est dans cet espace, dit Winnicott, que la créativité est possible et que nous sommes vivants en tant qu'être humain. «Cela fait place, dit-il, à la possibilité d'accepter la différence et la similarité [...]. Les phénomènes transitionnels représentent les premiers stades de l'utilisation de l'illusion sans laquelle l'être humain n'accorde aucun sens à l'idée d'une relation avec un objet perçu par les autres comme extérieur à lui » (Winnicott, 1971, 21). On pourrait penser que ce mouvement dialectique de la psyché est contemporain de l'apparition de la perspective et d'un début de relativité. Avec l'intériorisation de cet espace et de sa paradoxalité, l'enfant est prêt à concevoir l'extériorité.

\section{L'objet détruit-trouvé}

Au début, la mère a une fonction de holding, ensuite elle supervise le sevrage. Comme l'enfant la confond avec lui-même, il ne l'a pas encore vraiment remarquée comme distincte et extérieure. Maintenant que son besoin d'autonomie va grandissant, il souhaite s'affranchir de cette présence tutélaire. Il dépendra alors de la capacité de la mère à survivre à ses attaques, pour lui assurer l'accès à l'extériorité et à l'épreuve de réalité. "Cette activité destructrice, dit Winnicott, correspond à la tentative que fait le patient pour placer l'analyste hors du contrôle omnipotent, c'est-à-dire dehors dans le monde» (Winnicott, 1971, 127). C'est le paradoxe de «l'objet détruit/ trouvé». Lorsque l'enfant détruit l'objet subjectif pour s'en affranchir, voire s'en débarrasser, pour le mettre en dehors de lui, la mère qui survit est celle qui apparaît dans son extériorité et révèle du coup l'espace intérieur du fantasme. Le fantasme, dès lors, peut être considéré non plus comme un contenu mais comme un contenant. Désormais, la réalité psychique se distingue de la réalité extérieure et permet «la découverte d'un monde d'objets utilisables, avec qui entrer en relation dans un monde en dehors de soi » (Ogden, 1985b, 362). L'objet qui survit ne se dérobe pas dans le repli, la dépression, ou l'exercice de représailles. Mais, comme le dit Roussillon, encore faudra-t-il que le patient permette cette survie. 
Si par ailleurs l'objet ne survit pas, l'enfant se confondra dans l'objet subjectif tout-puissant. Il restera plus ou moins pris dans les rets de l'hallucinatoire, face à l'altérité qui sans cesse se dérobe et le mortifie. Emprisonné dans le monde magique du fantasme, il sera incapable de profiter des ressources du monde extérieur et sera seul pour assurer sa sécurité (Ogden 1985b, 362). "Le temps théorique de l'autodestruction, écrit Reid, est secondaire à une rencontre insuffisamment bonne entre psyché et environnement» $(2005,20)$.

Par ailleurs, si l'objet survit, le désinvestissement de l'objet subjectif, soit sa destruction, favorisera l'investissement ou le désir de l'objet externe et de sa représentation. La force du désir serait-elle cette contre-force à l'hallucinatoire évoquée par Reid, soit un contre-investissement (refoulement originaire?) qui assure désormais une limite entre inconscient et préconscient, de même qu'entre dedans et dehors? "Cette destruction, dit Winnicott, devient la toile de fond inconsciente de l'amour d'un objet réel; c'est-à-dire un objet en dehors de l'aire de contrôle omnipotent du sujet» (Winnicott, 1971, 131). Faut-il ajouter que l'objet trouvé hors du contrôle omnipotent est un objet total, séparé, sexué, ambivalent et de ce fait, bien souvent décevant. Il est le lieu d'une perte à l'orée de l'objet oedipien, sinon son contemporain. Il annonce la "position dépressive» théorisée par Mélanie Klein, et la possibilité du deuil.

Toute rupture traumatique de l'unité mère-enfant, comme « un coup de tonnerre» (Reid), brusquera le processus de désillusion, engendrant des angoisses paroxystiques. L'instauration de l'espace transitionnel sera mise en péril de même que la constitution de la fonction symbolique et de la capacité de pensée. Nous comprenons désormais que l'avènement du transitionnel, constitue l'acte fondateur pour la création d'un psychisme humain » (Reid, 2005, 38). De plus, Reid voit dans le paradoxe de l'objet détruit/trouvé l'actualisation d'un couple pulsionnel de première génération qu'il nomme créativité-destructivité, à l'instar du couple pulsionnel freudien pulsion de vie et pulsion de mort. Pour Danon-Boileau, qui dit «espace potentiel dit espace dépourvu de pulsion : la pulsion ne naît que de la distance entre sujet et objet; sitôt cette distance abolie, elle s'exténue » $(2007,244)$. Il faut peut-être considérer ce couple pulsionnel comme l'expression du paradoxe et se résigner à ce qu'il demeure vivant et non résolu.

\section{Clinique}

Sur le plan clinique, on retrouvera des organisations de survie que Joyce McDougall nomme pathologies de l'objet transitionnel (McDougall, 1978, 
155-156). Organisation-limite de la personnalité ou problématique narcissique-identitaire d'un sujet en mal d'espace, en mal d'être ou de naître, en mal d'identité et de pensée symbolisante. Un sujet qui signifiera de toutes les manières qu'il ne trouve pas sa place parmi les autres ou qui souhaitera, à son insu, être reconnu ou être mis à sa place. Enfin, toutes les constellations ou combinaisons sont possibles entre la quantité d'investissement de l'objet objectif et de l'objet subjectif. La nosologie est ici peu utile. Par exemple, surinvesti, l'objet fantasmatique/subjectif devient une chose concrète en soi, aussi puissant, dangereux ou gratifiant que la réalité matérielle dont il ne peut se différencier. C'est le monde schizoparanoïde, décrit par Mélanie Klein. On retrouve à l'autre extrême un surinvestissement de l'objet objectif ou de la réalité matérielle pour se protéger de la puissance de l'hallucinatoire comme chez les «surnormaux» décrits par Joyce McDougall $(1978,222)$, ou d'une autre manière, dans les troubles psychosomatiques avec la pensée opératoire et l'alexithymie qui les caractérisent. On pourrait aussi évoquer les patients souffrant de troubles autistiques qui, face à une angoisse insurmontable venue du monde, ont érigé un mur implacable.

Dans le cadre de la cure, «il s'agira moins de lever l'amnésie infantile, dira Green, que d'autoriser l'enfance à se constituer en mémoire fictionnelle » (cité par Reid, 2006, 1555). Dans tous les cas, l'analyste verra ses repères identitaires plus ou moins mis à l'épreuve, ce qui implique qu'il aura lui-même mené sa propre analyse aussi loin que possible, jusqu'à l'ébranlement de ses assises identitaires. C'est ce qui pourra assurer son aptitude à contenir l'angoisse et sa capacité négative, ce qui lui permettra de jouer justement, de se laisser jouer tout en restant à sa place de sujet symbolisant; particulièrement dans les situations d'impasse ou de suspens où... il n'y a pas grand espace de jeu et qui ne sont pas de tout repos... des situations parfois aux limites de l'analysable. C'est quand même par cette aptitude au jeu, «à la régression formelle de la pensée jusqu'aux abords de l'hallucinatoire », et paradoxalement, par un travail vigilant de contre-transfert, que viendra l'élan créateur à la base de l'interprétation dite mutative. Aptitude à «se laisser déporter ", disait Lise Monette, ou encore, à se mettre en résonance avec l'analysant, à l'écoute de la sensorialité des mots et du silence, de leur «livre de chair». Présenceabsence, "écoute flottante ", autant d'expressions qui expriment la dimension de l'Être. Sinon, toute interprétation risquera d'être perçue par l'analysant comme une résistance de l'analyste, et souvent à juste titre d'ailleurs. Wilfrid Reid qualifie ce type d'écoute de «transitionnelle», qui permet à l'analysant «de situer l'interprète dans un lieu autre que celui dévolu à 
l'objet pulsionnel afin qu'il puisse être conçu autrement qu'objet d'amour ou de haine» (Reid, 2006, 1554). Ce type d'écoute est spécifique à la méthode psychanalytique. «Acceptant ainsi de nous laisser atteindre de l'intérieur par notre patient », Guy Lavallée conclut avec pertinence, que «la psychanalyse est une colossale épreuve d'altérité» (Lavallée, 2005, 9). Le jeu le plus sophistiqué du vingtième siècle, écrivait Winnicott.

Concernant cette aptitude au jeu ou à l'invention, Danon-Boileau nous fait remarquer que la principale différence entre Winnicott et Freud réside dans leur capacité de jouer. Chez Freud, dit-il, «on ne joue pas par plaisir», "on joue pour maîtriser l'absence, ou le traumatisme». "On sublime pour les mêmes raisons, pour trouver une issue honorable à la pulsion.» "Chez Winnicott, au contraire on joue pour rien. Pas tout à fait même pour le plaisir, presque par désoeuvrement, ou plutôt pour retrouver la conviction qu'il existe en soi un espace potentiel qui n'est ni de soi ni de l'autre» (DanonBoileau, 2007, 244). Un «lieu de repos» dans cette tâche interminable qui consiste à maintenir à la fois séparées et reliées l'une à l'autre, réalité interne et réalité externe. Un lieu où se situe le jeu de l'enfant, l'activité créatrice et l'expérience culturelle (Winnicott 1971). Ce qui nous amène à la conception de l'expérience psychanalytique. Selon Kristeva, cette expérience est au cœur de l'œuvre de Mélanie Klein qui après avoir fait l'éloge du Sein de la Mère, en arrive à la fin de son œuvre, à l'éloge du matricide comme condition de pensée. Une Mère sans cesse à détruire dans le phantasme et à recréer dans la réalité (Kristeva, 2000, 189-225).

\section{Pour conclure}

Une troisième topique représente un outil qui rend possible l'élaboration d'une métapsychologie de l'espace, de l'unidimensionnalité à la tridimensionnalité: espace psychique du dedans, espace psychique du dehors et espace intermédiaire qui participe de l'un et de l'autre sans être ni l'un ni l'autre, et dont dépend notre capacité de penser. Un outil qui permet d'appréhender le fondement de nos intérieurs, notre espace psychique et la représentation que l'on s'en fait. Cette dernière évolue à travers les Âges. Ainsi, la Perspective, cette troisième dimension, existait déjà chez les Anciens grecs et réapparut dans la peinture à la Renaissance, après avoir traversé l'espace plat du Moyen-Âge qui l'avait occultée. Einstein, la physique quantique, le cubisme ont fait éclater l'espace Euclidien. Les notions d'Après-coup, de Hors-temps, d'Actuel ne cessent de résonner et de nous faire raisonner. Le temps psychique est une quatrième dimension et on pourrait penser à une 
métapsychologie de l'Espace-temps. Bion quant à lui, évoque un espace psychique pluridimensionnel. La métapsychologie de l'espace n'a pas fini d'être inventée/découverte. Sitôt qu'elle devient une théorie achevée, solipsiste, elle risque de se refermer sur elle-même et de perdre de vue son objet, soit l'espace et la notion d'infini qui lui est consubstantielle.

$\mathrm{Au}$-delà d'une troisième topique peut-être?

\author{
Lise Marceau \\ 1502, rue de la Citière \\ Québec \\ G1Y 1N5 \\ marceaul@videotron.ca
}

\title{
Note
}

1. Texte revu, présenté à la Société psychanalytique de Québec en novembre 2006 en guise d'introduction à une conférence de Wilfrid Reid sur la nécessité d'une troisième topique.

\section{Références}

Brusset, B., 2006, Métapsychologie des liens et troisième topique, Revue française de psychanalyse, $\mathrm{n}^{\circ} 5$ Spécial congrès, 1213-1283.

Botella, C. et Botella, S., 1990, La problématique de la régression formelle de la pensée et de l'hallucinatoire, in La psychanalyse pour demain. Monographies de la revue française de psychanalyse, Paris, Presses Universitaires de France, 63-90.

Botella, C. et Botella, S., 2002, Hallucinatoire, in Dictionnaire international de la psychanalyse, Paris, Calmann-Levy, 723-725.

Chabert, C., 2007, Texte de sa conférence au congrès de la SCP, inédit.

Danon-Boileau, L., 2007, De L'agir à la créativité, Revue française de psychanalyse, $\mathrm{n}^{\circ} 1$, Paris, Presses Universitaires de France, 233-245.

Freud, S., 1895, Esquisse pour une psychologie scientifique, in La naissance de la psychanalyse, Paris, Presses universitaires de France.

Freud, S., 1899, L'interprétation des rêves, Euvres complètes, tome IV, 1899-1900, Paris, Presses universitaires de France, 2004.

Freud, S., 1914, Pour introduire le narcissisme, Euvres complètes, tome XII, 1913-1914, Paris, Presses universitaires de France, 2005, 213-246.

Freud, S., 1923, Le Moi et le ÇA, Euvres complètes, tome XVI, 1921-1923, Paris, Presses universitaires de France, 1991, 255-302.

Green, A., 1980, Passions et destin des passions, in La folie privée, Paris, Gallimard, 1990, 140 193.

Green, A., 1993, Le travail du négatif, Paris, Les éditions de Minuit.

Kristeva, J., 2000, Le génie féminin, tome II, Mélanie Klein,, Paris, Fayard, 189-216.

Lavallée, G.., 2005, Régrédience, progrédience et hallucinatoire de transfert, texte publié sur le site web de la SPP.

Leclaire, S., 1981, Rompre les charmes. Recueil pour des enchantés de la psychanalyse, Paris, InterÉditions. 
McDougall, J., 1978, Plaidoyer pour une certaine anormalité, Paris, Gallimard.

Ogden, T., 1985a, On Potential space, Internationl Journal of Psychoanalysis, 66, 129-141.

Ogden, T., 1985b, The Mother, the Infant and the matrix: Interpretations of Aspects of the work of Donald Winnicott, Contemporaty Psychoanalysis, 21. 346-371.

Ogden, T., 2001, Reading Winnicott, Psychoanalytic Quarterly, 70, 299-323.

Pontalis, J.-B., 1977, Naissance et reconnaissance du « soi », in Entre le rêve et la douleur, Paris, Gallimard, 159-189.

Reid, W., 1997, Plaidoyer pour la monadologie freudienne ou pour en finir avec la légende d'un Winnicott antisexuel, in Revue française de psychanalyse, 61 (4), 1317-1342.

Reid, W., 2005, Au sujet du trauma ou quand le traumatisme devient traumatique, in Bulletin de la Société psychanalytique de Montréal, 17 (3).

Reid, W., 2006, De l'ensemble individu/environnement à la troisième topique: la pulsion, le narcissisme, l'emprise et la relation d'objet, in Revue française de psychanalyse, 70 (5), 1543-1557.

Winnicott, D.W., 1971, Jeu et réalité, Paris, Gallimard.

Winnicott, D.W., 1969, De la pédiatrie à la psychanalyse, Paris, Payot. 